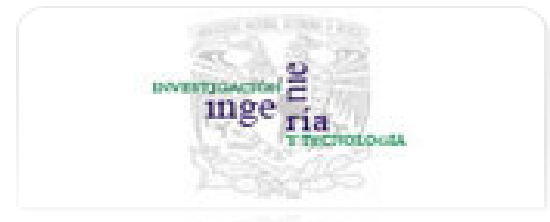

Ingeniería. Investigación y Tecnología

ISSN: $1405-7743$

iit.revista@gmail.com

Universidad Nacional Autónoma de México

México

Cerrud Sànchez, Sara Mercedes; Jacobo Armendáriz, Víctor Hugo; Ortiz Prado, Armando;

Schouwenaars, Rafael

Deterioro por corrosión atmosférica de componentes electrónicos de equipo telefónico

Ingeniería. Investigación y Tecnología, vol. VI, núm. 4, octubre-diciembre, 2005, pp. 219-238

Universidad Nacional Autónoma de México

Distrito Federal, México

Disponible en: http://www.redalyc.org/articulo.oa?id=40460401

- Cómo citar el artículo

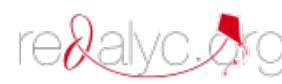

- Número completo

- Más información del artículo

Página de la revista en redalyc.org

Sistema de Información Científica

Red de Revistas Científicas de América Latina, el Caribe, España y Portugal Proyecto académico sin fines de lucro, desarrollado bajo la iniciativa de acceso abierto 


\title{
Deterioro por corrosión atmosférica de componentes electrónicos de equipo telefónico
}

\author{
Cerrud-Sánchez S.M. †, Jacobo-Armendáriz V.H., Ortiz-Prado A. y Schouwenaars R. \\ Departamento de Ingeniería Mecánica, Facultad de Ingeniería, UNAM \\ E-mails: cerrud@servidor.unam.mx,armandoo@servidor.unam.mx
}

(recibido: julio de 2004; aceptado: abril de 2005)

\begin{abstract}
Resumen
El deterioro del equipo electrónico por efectos ambientales, ha sido un fenómeno estudiado desde fines de la década de los 60 ; en general, se reconoce a éste como un fenómeno característico de atmósferas interiores. A la fecha, se han publicado diversos estudios que correlacionan las condiciones de exposición típicas de países industrializados con el deterioro y los mecanismos a través de los que éste se da. Dichos países, por su ubicación geográfica, tienen una climatología muy diferente a la de Latinoamérica. En este artículo, también se presentan los resultados del análisis de deterioro que, por exposición en campo, se observa en tarjetas telefónicas bajo diferentes condiciones climáticas de la República Mexicana. Asimismo, con base en los resultados se definen las peculiaridades del daño y los principales agentes que promueven la corrosión de estos equipos. Finalmente, se definen acciones tendientes a reducir los inconvenientes encontrados.
\end{abstract}

Descriptores: Corrosión atmosférica, análisis de falla, equipo electrónico.

\begin{abstract}
The fail ure de te rio ra tion of elec tronic de vices caused by at mo spheric cor ro sion has been stud ied since the late six ties. Most of the re search was ori ented to wards the climatologicconditionstypical forthein dustrialized countries. In these regions, the u se of air con di tion ing is very com mon. There fore, the differ ence be tween in door and outdoor at mo spheres is very im por tant. This sit u a tion is very differ ent in Latin Amer ica and the re search in this topic has been lim ited. In this pa per, are also pre sented the results of the fail ure anal $y$ sis in printed cir cuits from res iden tial tele phones. The electronic de vices have been in ser vice un der differ ent cli ma tic con di tions within Mexico. With the ob serva tions, it is pos sible to iden tify the main at mo spheric vari ables pro moting the cor ro sion in elec tronic de vices. The re sults are used to im prove the de sign of theelements.
\end{abstract}

Keywords: Atmosphericcorrosion, failure analy sis, electronic equip ment.

Introducción 
condiciones de los países industrializados (Guttenplan, 1987), (White et al., 1987), (Zakipour y Leygraf, 1986), (Rice et al., 1980), (Fukuda et al., 1991) y (Carrato et al., 1978). Sin embargo, la importancia que la electrónica tiene es innegable, razón por la que existe un porcentaje cada día creciente de equipo que entra en operación en países ca- talogados como economías emergentes.

Las condiciones climáticas en los países desarrollados (localizados, en general, por arriba del paralelo 30 de latitud Norte) son sensiblemente diferentes a las típicas de las economías emergentes (temperaturas máximas y mínimas, humedad relativa, precipitación fluvial); asimismo, los índices de contaminación (concen- traciones de $\mathrm{NO}_{x}, \mathrm{SO}_{2}$,
$\mathrm{CO}_{2}, \mathrm{H}_{2} \mathrm{~S}, \mathrm{NH}_{3}, \mathrm{O}_{3}$, entre otros) son, en gen eral, más agresivos en éstas. Los mayores índices de contaminación se presentan normalmente en mega ciudades, muchas de las cuales corresponden a países en vías de desarrollo (Tabla 1).

Dadas las variaciones climáticas características de las regiones ubicadas al Norte del paralelo 30, es común el empleo de sistemas de acondiciona- miento de aire, situación que atenúa sensiblemente la agresividad de las atmósferas interiores (Riceet al., 1980) y (Fukuda et al., 1991). Por su parte, en países como México no es extensivo el empleo de instalaciones de aire acondicionado, por lo que existe un importante intercambio de

Tabla 1. Índices gener ales de contaminación del aire en las 20 Ciudades más pobladas del planeta (UNEP/WHO, 1992)

\begin{tabular}{ccccc}
\hline & \multicolumn{3}{c}{ Calidad del aire en 20 ciudades } & \\
\hline Bangkok & $\mathrm{SO}_{2}$ & $\mathrm{CO}$ & $\mathrm{NO}_{2}$ & $\mathrm{O}_{3}$ \\
Beijing & 1 & 1 & 1 & 1 \\
Bombay & 3 & $\mathrm{NEDD}$ & 1 & $\mathrm{NEDD}$ \\
Buenos Aires & 1 & 1 & 1 & $\mathrm{NEDD}$ \\
Cairo & $\mathrm{NEDD}$ & $\mathrm{NEDD}$ & $\mathrm{NEDD}$ & $\mathrm{NEDD}$ \\
Calcuta & $\mathrm{NEDD}$ & 2 & $\mathrm{NEDD}$ & $\mathrm{NEDD}$ \\
Delhi & 1 & $\mathrm{NEDD}$ & 1 & $\mathrm{NEDD}$ \\
Jakarta & 1 & 1 & 1 & 2 \\
Karachi & 1 & 2 & 1 & $\mathrm{NEDD}$ \\
Londres & 1 & $\mathrm{NEDD}$ & $\mathrm{NEDD}$ & 1 \\
Los Angeles & 1 & 2 & 1 & 3 \\
Manila & 1 & 2 & 2 & $\mathrm{NEDD}$ \\
Cd. de México & 1 & $\mathrm{NEDD}$ & $\mathrm{NEDD}$ & 3 \\
Moscú & 3 & 3 & 2 & $\mathrm{NEDD}$ \\
Nueva York & $\mathrm{NEDD}$ & 2 & 2 & 2 \\
Río de Janeiro & 1 & 2 & 1 & $\mathrm{NEDD}$ \\
Sao Paulo & 2 & 1 & $\mathrm{NEDD}$ & 3 \\
Seúl & 1 & 2 & 2 & 1 \\
Shanghai & 3 & 1 & 1 & $\mathrm{NEDD}$ \\
Tokio & 2 & $\mathrm{NEDD}$ & 1 & 3 \\
\hline
\end{tabular}

Contaminación: Muy severa (3), Severa (2), Moderada (1), No existen datos disponibles (NEDD). 
Cerrud-Sánchez S.M. †, Jacobo-Armendáriz V.H., Ortiz-Prado A. y Schouwenaars R.

aire con el ex te rior, situación que se tra duce en características mucho más agresivas de la atmósfera (en interiores) en la cual se encuentra instalado el equipo electrónico.

Todas estas circunstancias dan como resultado que muchas de las conclusiones y definiciones desarrolladas para la instalación de equipo y dis- positivos electrónicos en países industrializados, no apliquen para las situaciones características de países como México, por ello, es necesario realizar los estudios que permitan definir el deterioro característico para las condiciones medio ambientales de las diversas regiones de la República Mexicana.

En el caso del estudio de deterioro que por efecto del medio ambiente sufre el equipo electrónico, los métodos basados en la presencia de algunos contaminantes para la determinación de la agre- sividad del medio, no necesariamente son la mejor opción para definir la corrosión que sufre el equipo. Por lo tanto, es necesario definir meto- dologías idóneas que permitan determinar las peculiaridades del deterioro, los principales con- taminantes, el efecto que tienen las características constructivas del equipo, así como los dispositivos que mayor daño presentan.

Considerando lo anterior, se definió la conveniencia de estudiar un dispositivo o equipo estándar, el cual pudiera estar instalado bajo las condiciones más diversas, de preferencia, que fuera de un mo- delo específico y producido por

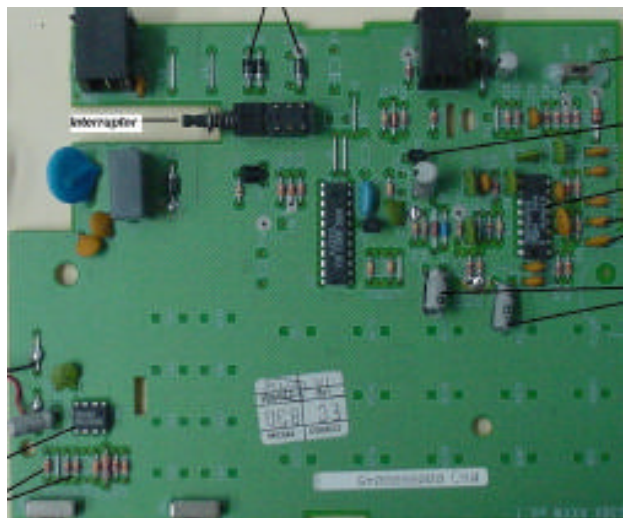

Figura 1. Tarjeta impresa típica de aparatos telefónicos de uso residencial una planta de re- ferencia. Con esto se pudo garantizar la consis- tencia de los patrones empleados para el estudio. Así, se definió que un espécimen que cumple con las condiciones señaladas son las tarjetas electrónicas de teléfonos de uso residencial (Figura 1).

Una vez determinado el elemento patrón, se procedió a precisar las localidades para obtener las muestras del estudio. Para esto, se consideró la clasificación climática (García, 1988), (Figura 2), el mapa de corrosividad atmosférica (Mariaca et al., 1999), los datos de las estaciones de monitoreo del aire publicados en INE-CENICA-JICA (1997) y los datos del INEGI (2003). Además, se tomaron en cuenta los parámetros que mayor influencia tienen en la corrosión atmosférica (Mariaca et al., 1999), (Zakipour et al., 1997) y (Oesh y Faller, 1997) y en particular, en el deterioro de equipo electrónico (Guttenplan, 1987), (White et al., 1987), (Rice et al., 1980), (Yukonovich, 2001), (Gouda et al., 1989), (Abbott, 1989), determinándose las localidades de referencia (Tabla 2).

Con la intención de garantizar la representatividad de los datos obtenidos en el estudio, considerando el grado de variabilidad en la exposición al interior del grupo de especimenes provenientes de una localidad y buscando optimar tiempo y costo del proyecto, se determinó que el tamaño de la muestra por localidad fuera de 5 a 7 aparatos telefónicos.

\section{Procedimiento para la obtención de muestras}

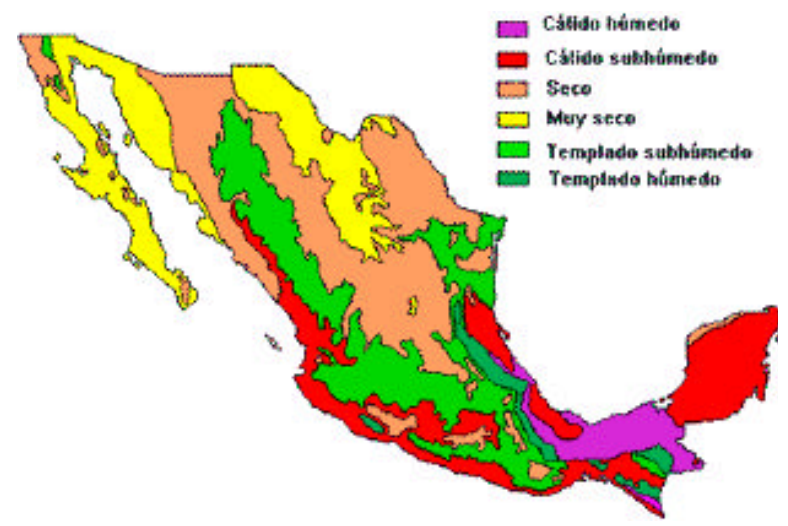

Figura 2. Distribución climática en la república mexicana [UNEP-WHO, 1992] 
Deterioro por corrosión atmosférica de componentes electrónicos de equipo telefónico

Tabla 2. Características climatológicas de las zonas geográficas para las cuales se analizó el deterioro de teléfonos de uso residencial

\begin{tabular}{|c|c|c|c|c|}
\hline Localidad & $\begin{array}{c}\text { Ubicación } \\
\text { (UNEO-WHO, 1992) }\end{array}$ & $\begin{array}{l}\text { Características } \\
\text { climatológicas } \\
\text { (Garcia, 1998) }\end{array}$ & Tipo de atmósfera & $\begin{array}{l}\text { Contaminantes } \\
\text { presentes }\end{array}$ \\
\hline $\begin{array}{c}\text { Área Metropolitana de } \\
\text { la Ciudad de México. } \\
\text { Se trata de una de las } \\
\text { zonas urbanas más } \\
\text { contaminadas del } \\
\text { planeta. }\end{array}$ & $\begin{array}{l}19^{\circ} 23^{\prime} \text { latitud Norte, } \\
99^{\circ} 10^{\prime} \text { longitud Oeste }\end{array}$ & $\begin{array}{c}\text { Dada la extensión } \\
\text { territorial de la zona } \\
\text { metropolitana de la } \\
\text { Ciudad de México, a } \\
\text { ésta se asocian } \\
\text { diversos microclimas, } \\
\text { los cuales van del BS } \\
\text { (seco o árido) al } \\
\text { Cb(w) (templado } \\
\text { subhúmedo con lluvia } \\
\text { en verano. } \\
\text { Temperatura } \\
\text { promedio } 16.5^{\circ} \text {, con } \\
\text { precipitaciones que } \\
\text { van de } 570 \text { a } 1100 \\
\text { mm } \\
220 \text { a } 2400 \text { m SNM } \\
\text { Humedad relativa } \\
\text { variable con la } \\
\text { estación (33 a 89). }\end{array}$ & $\begin{array}{c}\text { De acuerdo a las } \\
\text { zonas y considerando } \\
\text { las fuentes emisoras, } \\
\text { este concepto } \\
\text { también varia, pero } \\
\text { en general, se puede } \\
\text { definir como Urbano. } \\
\text { La principal zona de } \\
\text { generación de } \\
\text { contaminantes se } \\
\text { encuentra en el } \\
\text { Norte, con vientos } \\
\text { dominantes N, NW, } \\
\text { S, SE dependientes } \\
\text { de la estación }\end{array}$ & $\begin{array}{c}\text { Se trata de una de las } \\
\text { ciudades más } \\
\text { contaminadas del } \\
\text { planeta, en general, } \\
\text { las concentraciones } \\
\text { de } \mathrm{NO}_{\mathrm{x}}, \mathrm{CO}_{2}, \text { y SO } \\
\text { son importantes. }\end{array}$ \\
\hline $\begin{array}{c}\text { Cocoyoc-Oaxtepec, } \\
\text { Morelos }\end{array}$ & $\begin{array}{l}18^{\circ} 54^{\prime} \text { latitud Norte, } \\
98^{\circ} 58^{\prime} \text { longitud Oeste }\end{array}$ & $\begin{array}{c}\text { C(w) templado } \\
\text { subhumedo, con } \\
\text { lluvias en verano. } \\
\text { Temperatura } \\
\text { promedio anual } \\
21.9^{\circ} \mathrm{C} \text {, precipitación } \\
900 \text { mm } \\
1385 \text { m SNM. }\end{array}$ & Rural & $\begin{array}{c}\text { Propio de las } \\
\text { prácticas agrícolas } \\
\text { características de la } \\
\text { región. }\end{array}$ \\
\hline Cuernavaca, Morelos & $\begin{array}{l}18^{\circ} 55^{\prime} \text { latitud Norte, } \\
99^{\circ} 44^{\prime} \text { longitud Oeste }\end{array}$ & $\begin{array}{c}\text { Se presentan dos } \\
\text { microclimas, } \\
\text { templado subhúmedo } \\
\text { con lluvias en verano } \\
\text { C(w2), parte Norte y } \\
\text { semicálido } \\
\text { subhúmedo con } \\
\text { lluvias en verano } \\
\text { C(w1). } \\
20.7^{\circ} \mathrm{C}, 1146 \mathrm{~mm} \text {. } \\
1529 \text { m SNM. }\end{array}$ & Urbano & $\begin{array}{l}\text { Con la presencia de } \\
\text { contaminantes } \\
\text { característicos de las } \\
\text { fuentes móviles }\end{array}$ \\
\hline $\begin{array}{c}\text { Bahía de Banderas } \\
\text { (Estados de Jalisco y } \\
\text { Nayarit) }\end{array}$ & $\begin{array}{c}20^{\circ} 37^{\prime} \text { latitud Norte, } \\
105^{\circ} 14^{\prime} \text { 'longitud } \\
\text { Oeste }\end{array}$ & $\begin{array}{c}\text { A(w1), caliente } \\
\text { subhúmedo con } \\
\text { lluvias en verano, } \\
\text { Temperatura media } \\
\text { anual } 26^{\circ} \mathrm{C} \\
\text { precipitación pluvial } \\
1430 \mathrm{~mm} \\
2 \mathrm{~m} \text { SNM. }\end{array}$ & Marino & $\begin{array}{c}\text { Contaminantes } \\
\text { característicos de la } \\
\text { costa (Cloruros), } \\
\text { adicional a lo cual'se } \\
\text { considera la } \\
\text { presencia de } \\
\text { productos de la } \\
\text { actividad agrícola. }\end{array}$ \\
\hline $\begin{array}{c}\text { Coatzacoalcos, } \\
\text { Veracruz }\end{array}$ & $\begin{array}{c}18^{\circ} 09 ? \text { L Latitud Norte, } \\
94^{\circ} 25^{\prime} \text { Longitud } \\
\text { Oeste }\end{array}$ & $\begin{array}{c}\text { Condiciones Am } \\
\text { caliente húmedo con } \\
\text { lluvias en verano, las } \\
\text { cuales son muy } \\
\text { próximas al Af } \\
\text { Caliente humedo con } \\
\text { lluvias todo el año. } \\
25.4^{\circ} \mathrm{C} \text { de } \\
\text { temperatura media } \\
\text { anual, precipitación } \\
2780 \mathrm{~mm}, 14 \mathrm{~m} \text { SNM }\end{array}$ & Marino-Industrial & $\begin{array}{c}\text { Las condiciones de } \\
\text { salinidad en } \\
\text { combinación con la } \\
\text { elevada húmeda } \\
\text { relativa y las } \\
\text { emisiones producto } \\
\text { de la industria } \\
\text { petroquímica ahí } \\
\text { instalada, } \\
\text { transforman a este } \\
\text { punto en uno de los } \\
\text { de mayor índice de } \\
\text { corrosividad } \\
\text { atmosférica en el } \\
\text { país. }\end{array}$ \\
\hline
\end{tabular}


Cerrud-Sánchez S.M. †, Jacobo-Armendáriz V.H., Ortiz-Prado A. y Schouwenaars R.

Definidas las localidades se procedió a la selección de las muestras, en general, se utilizaron tarjetas provenientes de teléfonos en servicio que estuvie- ran instalados en casa habitación y alejados de las zonas de elevada humedad relativa (baños) y conta- minantes (cocina). En todos lo casos, se requirió conocer el tiempo de servicio, de tal forma que éste fuera inferior a 5 años. Los menores tiempos de exposición se tuvieron para los aparatos instalados en zonas costeras, particularmente, los aparatos provenientes de Tampico que mostraron fallas intermitentes, lo cual provocó que fueran retirados de servicio y almacenados. Es conveniente men- cionar que estos aparatos seguían en operación previo a su inspección. En general, las muestras permanecieron en almacenaje antes de su estudio en períodos menores a un mes, cuidando que durante el almacenaje no influyera el ambiente en los resultados (recipientes cerrados con presencia de deshumidificadores).

\section{Metodología para el análisis del deterioro}

En la figura 3 se presenta la metodología seguida para la determinación del deterioro de las tarjetas electrónicas, ésta consistió en:
1. Inspección de las tarjetas de $1 \mathrm{X}$ a 30X (estereomacroscopio), con la finalidad de determinar diferencias en el grado de de- terioro en los diversos componentes, permi- tiendo así la construcción de mapas de corrosión o ataque.

2. Determinación de zonas críticas y to- ma de muestra de dispositivos.

3. Inspección de elementos que presen- tan un marcado deterioro, esto mediante microscopia electrónica de barrido (SEM).

4. Caracterización de los depósitos for- mados mediante espectroscopía de rayos $X$ (EDS). Lo ante rior, permitió definir los con- taminantes críticos para cada condición y dispositivo (conectores, interruptores, ba- tería, potenciómetros). Esta técnica, si bien resultó muy demandante en tiempo y re- cursos de microscopia electrónica, permitió definir con relativa precisión los contami- nantes que mayor efecto tienen en el de- terioro del equipo.

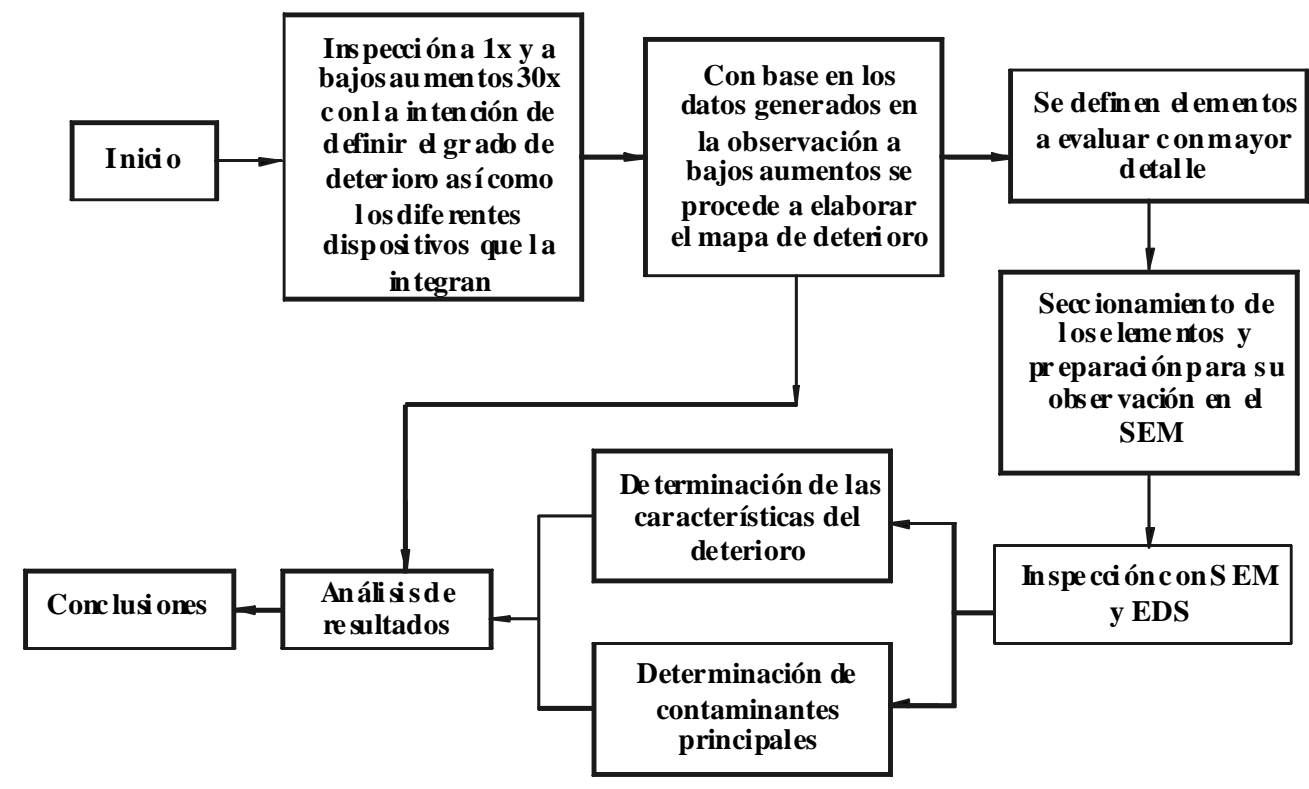

Figura 3. Metodología de estudio de tarjetas electrónicas de equipo telefónico de uso residencial 
El análisis de las tarjetas y la determinación de las características del deterioro, permiten, apli- cando la metodología de análisis de falla (Cerrud et al., 2002), proponer mejoras que garanticen una disminución del daño, a la vez de definir las condiciones atmosféricas críticas para el equipo electrónico.

Los trabajos publicados, normalmente refieren la corrosividad del ambiente, tanto en exteriores como en interiores, a partir de prue- bas de larga duración realizadas en laminillas metálicas (cupones). Sin embargo, las condi- ciones en que se desarrollan los mecanismos de corrosión en éstas poco o nada tienen que ver con lo que sucede al nivel de un dispositivo o tarjeta electrónica, para esto, se requiere del estudio de dichos elementos para así generar conclusiones que sean directamente aplicables a su deterioro por corrosión.

La problemática del deterioro ambiental en equipo electrónico se debe, además de la compleja interacción de los factores que en ésta intervienen, a que en la mayoría de los casos, no existe ninguna documentación causa-efecto, si- tuación que en el análisis presentado es detallada.

\section{Procedimiento para la elaboración de los mapas de deterioro}

Para facilitar la interpretación de los resultados se diseñaron mapas de deterioro (Figura 4), definién- dose cinco niveles de ataque en los componentes:

1. Depósito ligero de solución o de sales sin evidencia de corrosión.

2. Depósito importante de solución o de sales sin evidencia de corrosión.

3. Leve evidencia de corrosión.

4. Presencia de productos de corrosión, abundantes picaduras, presencia indiscu- tible de fenómenos corrosivos.

5. Daños importantes debidos a la corro- sión, la integridad de los elementos es afectada en forma notoria.

Los niveles 1 y 2 no significan la existencia de daños por corrosión, pero sí representan una situación previa al ataque, del nivel 3 al 5

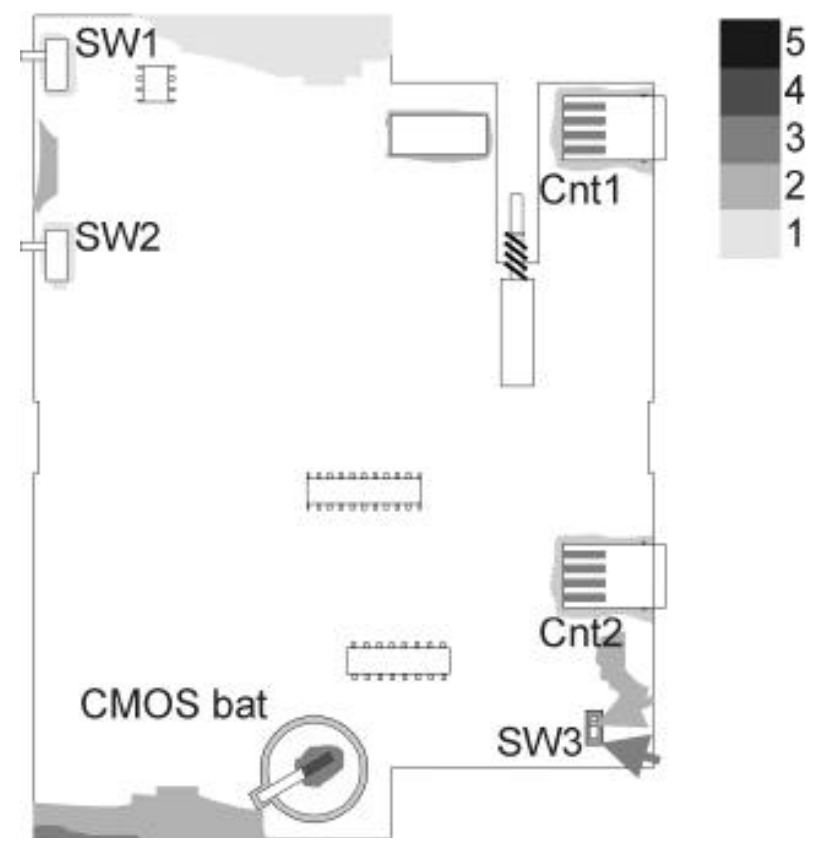

Figura 4. Mapa de deterioro típico para una tarjeta impresa de un teléfono de uso residencial 
existe corrosión, representando este último una condición inminente de falla.

\section{Resultados del análisis del deterioro observado en tarjetas y dispositivos electrónicos}

La tarjeta electrónica de un teléfono consiste de un circuito impreso típico al que están ensamblados diversos componentes (microcircuitos, resistencias, inductancias y capacitancias). En estos dispositivos se encuentran presentes una serie de metales y aleaciones ( $\mathrm{Cu}$, Al, latón, etc), así como diversos recubrimientos ( $\mathrm{Sn}, \mathrm{Au}$, $\mathrm{Ni}, \mathrm{Ag}$ ), lo que es característico de los equipos elec- trónicos.

Dadas las limitaciones inherentes al empleo del EDS, se analizó en los productos de corrosión la presencia de compuestos de azufre, cloro y oxígeno. El efecto del ozono y los óxidos de nitrógeno (NOx) no pudieron ser comprobados.

\section{Resultados de la inspección de las tarjetas electrónicas}

\section{Análisis de las muestras provenientes de Tampico}

De la inspección se observó:

a) Los mapas de deterioro son coincidentes.

b) Las tarjetas presentan marcas características al escurrimiento de líquido.

Los patrones de escurrimiento son similares a lo que se podría observar si en las tarjetas se derramara una solución acuosa (Figura 5). Dado que los teléfonos bajo estudio eran de diferente procedencia, se concluye que la posibilidad de que éstos hubieran sufrido el derrame de algún líquido no existe. Por lo an te rior, los patrones observados sólo pueden ser producidos por escurrimiento del condensado que se forma en la tarjeta durante su servicio, situación que a su vez, provoca pro- blemas de operación (razón de su retiro).

La formación de condensado representa la primer etapa del proceso corrosivo, por lo que se puede afirmar que la presencia de fallas, producto del deterioro, era solo cuestión de tiempo.

Del análisis detallado de las tarjetas se tiene:

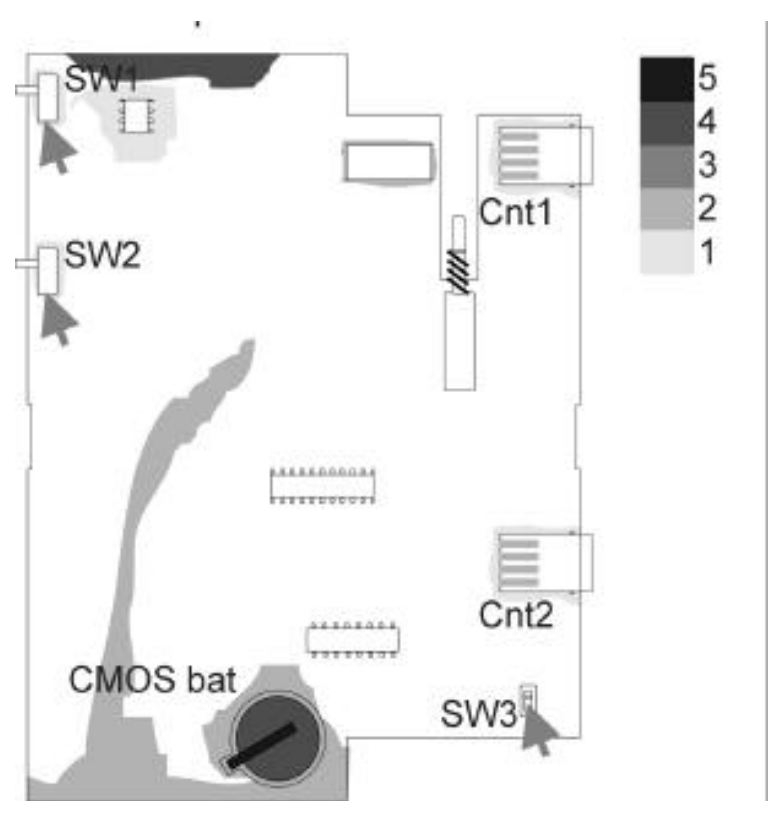

Figura 5. Mapa de tarjeta proveniente de Tampico. SW (interruptores), CMOS bat (batería de la memoria del teléfono) y CNT (contactos de cobre recubiertos con níquel, y con un recubrimiento 
a) En los interruptores (Figura 6) se combinan una celda galvánica con una zona de acumulación de agua. Aquí existe una reducida separación entre la carcaza (acero cromado) y el botón del interruptor. El elec- trolito se acumula fácilmente en el agujero de la carcaza, generando una celda entre el cromo del recubrimiento y el propio acero, dando lugar al deterioro de la caja pero no de las partes interiores del interruptor. La solución propuesta a este inconveniente re- side en la eliminación del cromado de la parte, o en su defecto, la sustitución por una pieza de plástico.

b) Se comprueba que al interior del aparato, existen gradientes de contaminación y que el empaque puede prevenir la corrosión de piezas sensibles, aun cuando éste no sea totalmente hermético.

c) Otro ejemplo importante de la corrosión galvánica se produce por el empleo de recubrimientos de oro. Dado que este ele- mento es el metal más noble, forma celdas galvánicas en contacto con cualquier otro metal. Se observó que la corrosión por debajo de los recubrimientos de oro en combinación con substratos de níquel y cobre es usual, dado que el oro aplicado de manera elec- trolítica tiende a ser poroso. Esta observación confirma los resultados de otros estudios publicados (Guttenplan, 1987), (Yukonovich, 2001). Estos mismos efectos se detectaron en especimenes sometidos a diferentes carac- terísticas atmosféricas.

d) Otro inconveniente se presenta en el broche de sujección de la pila de la memoria. En este caso, se manifiesta deterioro entre éste y la jaula de Faraday del aparato. El problema se debe a la combinación de un diseño desafortunado (en cuanto a la geo- metría) del elemento de sujección de la pila de la memoria, con la alta humedad relativa característica de la zona (Figura 7). En este caso, la forma

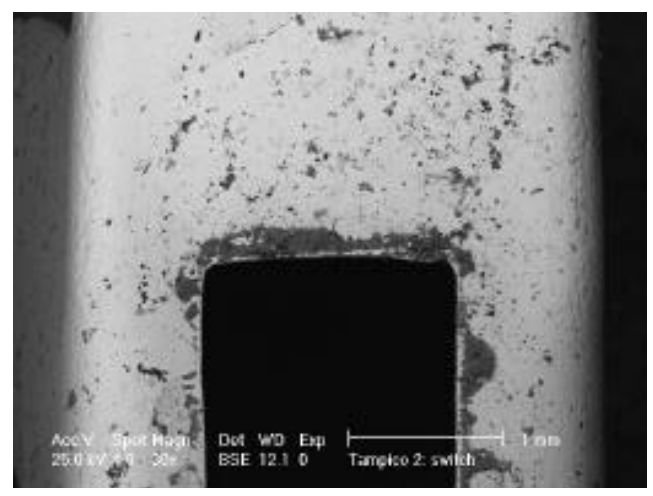

Figura 6. Carcaza de interruptor, es evidente la existencia de depósitos provocados cuando el electrolito se acumula en la zona troquelada, dando lugar a la presencia de un par galvánico

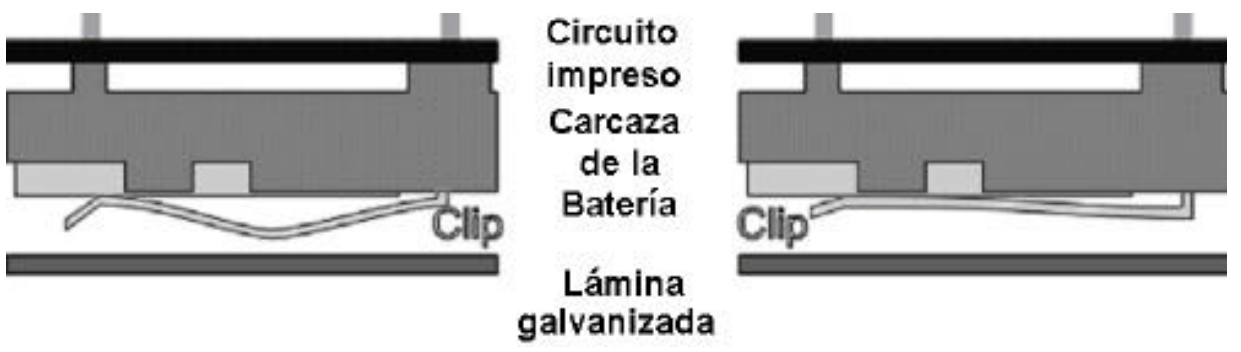

Figura 7. Se puede apreciar el diseño orig inal (a) del sujetador de la batería de memoria del teléfono. La curvatura del clip favorece la presencia de condensado y provoca corrosión galvánica entre el recubrimiento del clip (estaño), y el de la lámina (zinc). Con una modificación (b) en la pieza 
del clip (Figura 7a) favorece la presencia de condensado, el cual en com- binación con la diferencia de materiales del broche (acero estañado), de la carcaza de la pila (acero inoxidable) y de la jaula de Faraday (acero galvanizado), se traduce en un rápido deterioro de la lámina galvanizada, así como inconvenientes en la carcaza de la propia batería. Una posible solución del problema se presenta en la figura $7 b$, en la cual se propone modificar la curvatura del broche para evitar la formación de condensado.

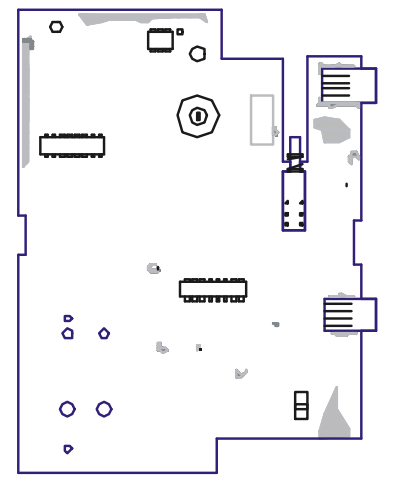

Figura 8. Se muestra la distribución de los depósitos, estrictamente no se evidenció daño. El aparato corresponde a la zona poniente del área metropolitana de la Ciudad de México

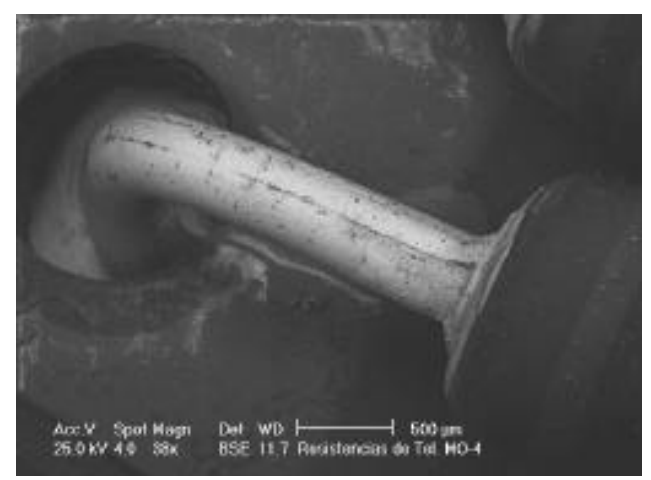

Figura 9. En el conductor de esta resistencia se aprecia que el recubrimiento de estaño se encuentra prácticamente sin deterioro. Los depósitos corresponden a óxidos

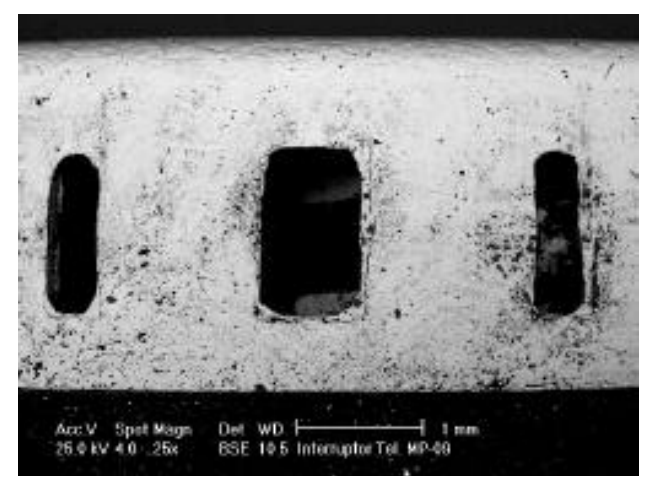

Figura 10. Interruptor. Se trata de una lámina de acero de bajo carbono troquelada y niquelada. La formación de depósitos también en este caso es mínima 
Deterioro por corrosión atmosférica de componentes electrónicos de equipo telefónico

\section{Análisis de las muestras provenientes del área metropolitana de la Ciudad de México}

Esta región representa una de las zonas de menor calidad del aire, no solamente en México sino en el mundo, por tal motivo, los resultados corres- pondientes en principio son sorprendentes, ya que fue relativamente difícil encontrar evidencia de corrosión en los diversos componentes de las tarjetas (Figuras 8, 9 y 10). La contaminación en la Ciudad de México es sin duda muy severa; sin embargo, los principales problemas son el ozono, los óxidos de nitrógeno (NOx), los compuestos orgá- nicos volátiles (VOCs) y partículas suspendidas. Es conocido que todas estas sustancias aceleran la corrosión en presencia de otros contaminantes. $\mathrm{El} \mathrm{SO}_{2}$ está presente, mientras que se puede suponer que el cloro juega un papel de menor importancia. La diferencia principal, desde el punto de vista del ataque observado en las tarjetas electrónicas, entre Tampico y la Ciudad de México es la humedad. En Tampico, la condensación nocturna es lo suficientemente intensa para provocar la falla en sistemas electrónicos en una etapa incipiente de corrosión. Este fenómeno es inexistente en la Ciudad de México. La remoción de $\mathrm{NOx}$ y ozono en forma de $\mathrm{HNO}_{3}$ y $\mathrm{H}_{2} \mathrm{O}_{2}$ necesita la presencia de una fase acuosa, por lo que su influencia en la región de Tampico será más fuerte. La acumu- lación de $\mathrm{SO}_{2}$ sigue la misma lógica, con excepción de la plata, la cual se corroe en atmósferas secas.

Por consecuencia, se puede concluir que la baja humedad relativa característica de la zona me- tropolitana de la Ciudad de México se traduce en mucho menores índices de deterioro en el equipo electrónico, si bien para esto habrá que considerar la existencia de microclimas en la propia zona metropolitana.

\section{Análisis de las muestras provenientes de la Región Oaxtepec-Cocoyoc}

La corrosividad característica de esta región se pre sume, en principio, que debe ser menor que la correspondiente para Tampico y la Ciudad de México, esto en virtud de ser una zona con reducida contaminación atmosférica, altas tem- peraturas y precipitaciones medias. Sin embargo, las observaciones realizadas demuestran una importante precipitación de compuestos de azu- fre, lo cual es contradictorio a las condiciones esperadas de contaminación, dado que se trata de una atmósfera rural. Por otra parte, resulta también sorprendente la presencia de altos niveles de cloruros. Los análisis realizados demuestran que el cloro está combinado con potasio, además de que las partículas de hollín eran abundantes en todas las muestras inspeccionadas. Estos contaminan- tes se pueden correlacionar con prácticas agrí- colas de las regiones adyacentes, en las que muchas de las tierras de cultivo disponibles se orientan a la siembra de caña de azúcar, campos agrícolas que antes de la zafra son quemados. Esto da lugar a la presencia de compuestos de cloro y a la alta incidencia de partículas de carbón. Otro contaminante presente en niveles mayores que en las otras zonas, son las partículas, asimismo, se determinó la presencia de Al, Si y Ca en los productos de corrosión.

El deterioro observado en las muestras provenientes de Cocoyoc se concentra generalmente alrededor de partículas o sales cristalizadas, esto es una indicación de una condensación limitada e intermitente en las superficies, mientras que los contaminantes se acumulan durante la fase húmeda y se cristalizan al secarse. La presencia de sales higroscópicas o partículas de polvo que sirven como inoculantes, causan la condensación en el mismo lugar durante cada ciclo de condensación-secado. Lo an te rior con duce a cuestionar la aseveración de que las zonas rurales no están contaminadas, situación que no puede ser aceptada sin un análisis crítico. Asimismo, en es- tas muestras se observaron evidencias de corrosión a través del recubrimiento de oro (no homogéneo), en alambres de cobre con reves- timiento intermedio de níquel (Figura 11). Los productos de corrosión se caracterizan por la presencia de depósitos de cloruros de níquel y cobre, combinados con compuestos de azufre en menor proporción. En la figura 12 se observa el deterioro de un conector de latón, el cual se encontraba en contacto con un alambre revestido con oro. En este caso, se presenta la 
Cerrud-Sánchez S.M. †, Jacobo-Armendáriz V.H., Ortiz-Prado A. y Schouwenaars R.

formación de compuestos del sustrato, los cuales fluyen a través de los poros del recubrimiento de oro. Es evidente que también existe la formación de una celda galvánica al entrar en contacto el conector de latón con el alambre dorado, situación que favorece la corrosión del latón, dando como resultado la presencia de productos de corrosión de cobre y zinc, en los que se manifestó también la existencia de cloro y cantidades menores de

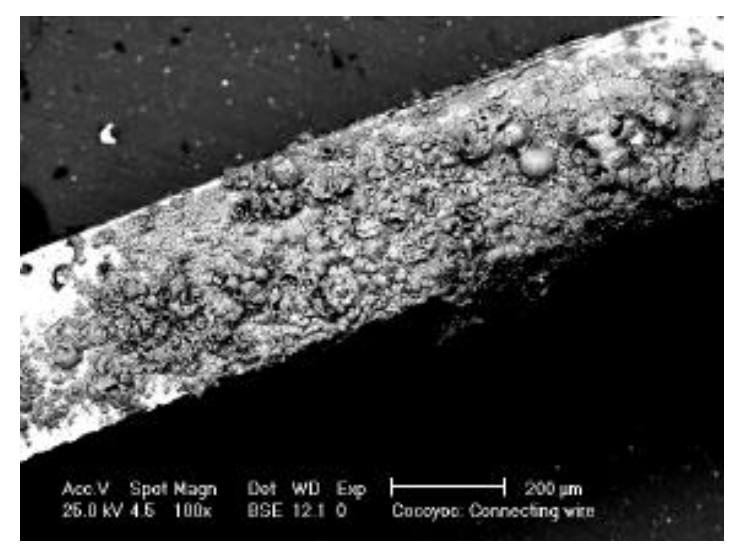

Figura 11. Productos de corrosión en un alambre de cobre recubierto con níquel y con un depósito ex te rior de oro

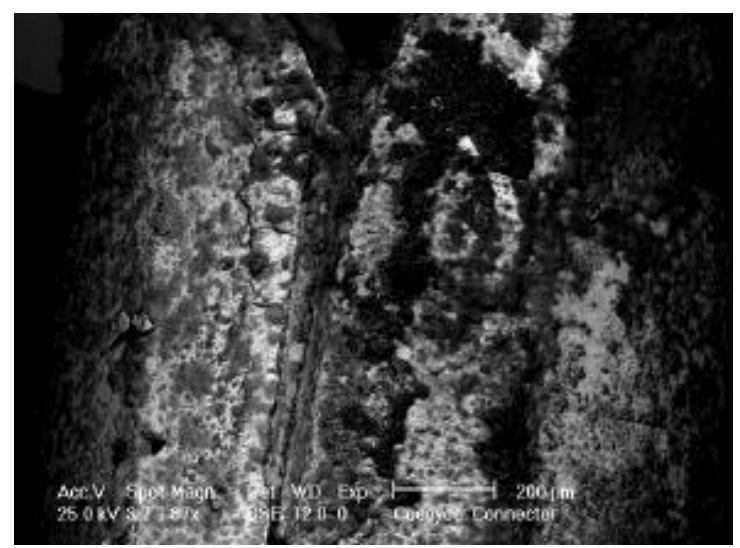

Figura 12. Conector de latón en contacto con un alambre recubierto con oro. En general, la superficie se ve deteriorada por efecto de la corrosión (zonas oscuras). Los productos de corrosión se caracterizan por la presencia de cloro, y en menor proporción azufre

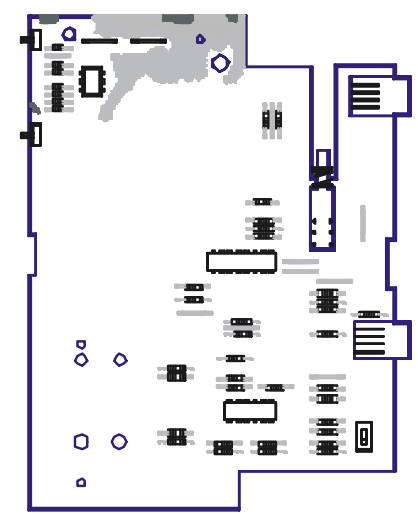


Deterioro por corrosión atmosférica de componentes electrónicos de equipo telefónico

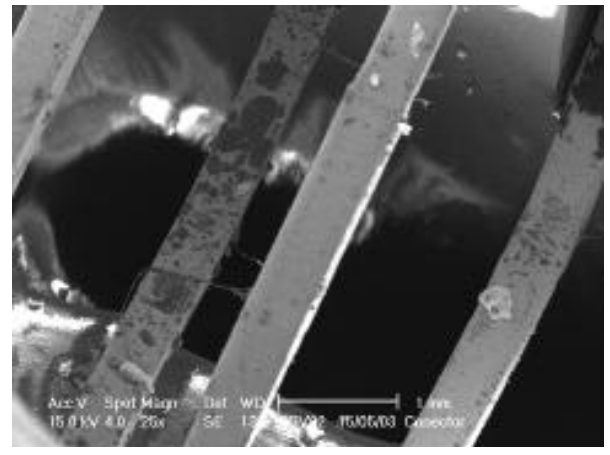

Figura 14. Pines de conexión de cobre recubiertos de oro. Se manifiesta la formación de depósitos

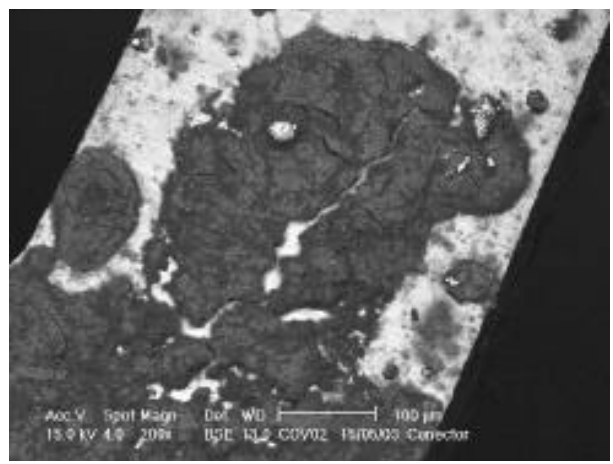

Figura 15. A través de las heterogeneidades del recubrimiento de oro, se lleva a efecto el ataque de la capa intermedia de níquel y del propio cobre del conductor

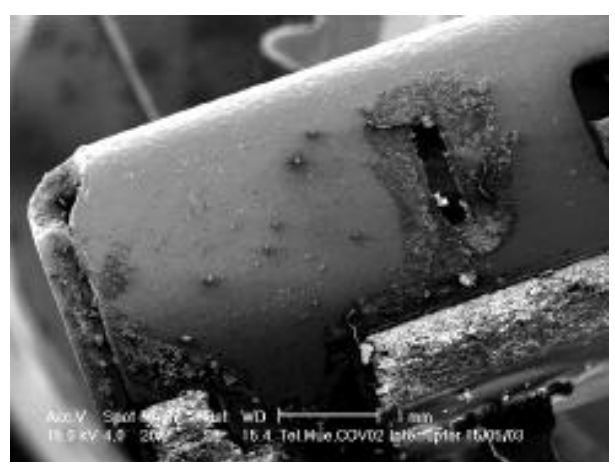

Figura 16. Imagen a bajos aumentos $(20 x)$ de la carcaza del interruptor. Se trata de acero de bajo carbono niquelado

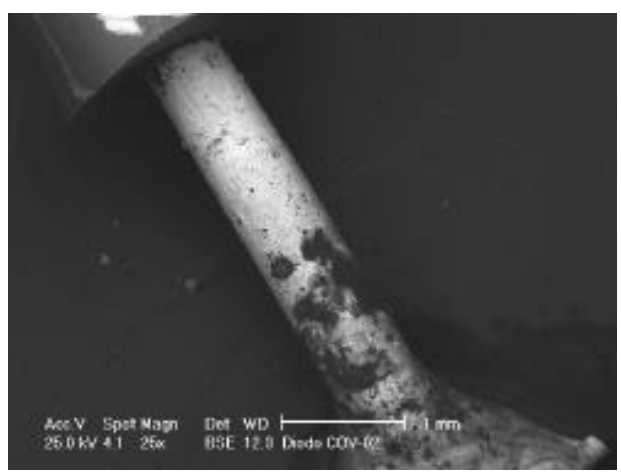

Figura 17. Conector de diodo (25x). Se trata de un alambre de cobre estañado 
Cerrud-Sánchez S.M. †, Jacobo-Armendáriz V.H., Ortiz-Prado A. y Schouwenaars R.

azufre combinado con oxígeno. Lo anterior, confirma la teoría de que el uso del oro promueve la corrosión en equipo electrónico, en lugar de prevenirla, esto en función de los contaminantes presentes y de las condiciones de exposición.

\section{Análisis de las muestras provenientes de Coatzacoalcos, Veracruz}

Se puede considerar que una de las regiones más críticas en México, desde el punto de vista de corrosión atmosférica, es la zona de Coatzacoalcos, ya que en ésta se conjugan la mayoría de las variables que mayor efecto tienen en dicho fenómeno (elevada humedad y temperaturas, contaminantes producto de las emisiones de la industria pesada y de fuentes móviles, cloruros, etc.). Sin embargo, las observaciones realizadas indican en general, grados de deterioro inferiores a los reportados para los especimenes provenientes de Tampico. Esto se debe a que las muestras provenientes de Tampico estaban instaladas en casas habitación sin aire acondicionado, por su parte, las condiciones climáticas más extremas de Coatzacoalcos dan como consecuencia que los equipos se encontraran en ambientes con acon- dicionamiento de aire, situación que re duce los problemas de acuerdo a lo observado en las tarjetas (Figuras 13 a 17). Asimismo, es con- veniente mencionar que los equipos retirados de esta localidad estuvieron en operación du rante mucho mayor tiempo (2 a 4 años) que los de Tampico (3 a 12 meses). Esta situación confirma la importancia que un ambiente con menor inter- cambio de aire con el exterior, humedad contro- lada y sin importantes variaciones de temperatura (mínima condensación) tiene en el deterioro del equipo electrónico. En los equipos retirados de Coatzacoalcos existe mínima evidencia de for-

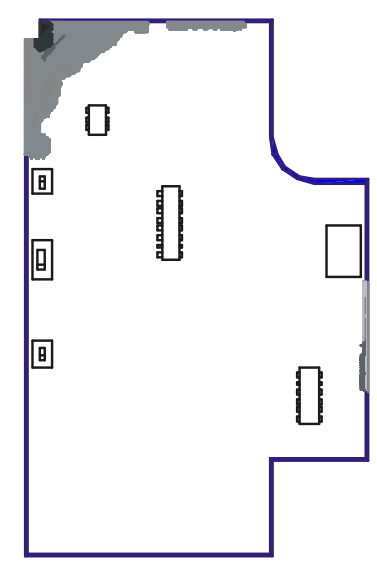

Figura 18. Mapa del deterioro de una tarjeta impresa correspondiente a un aparato instalado en Puerto Vallarta

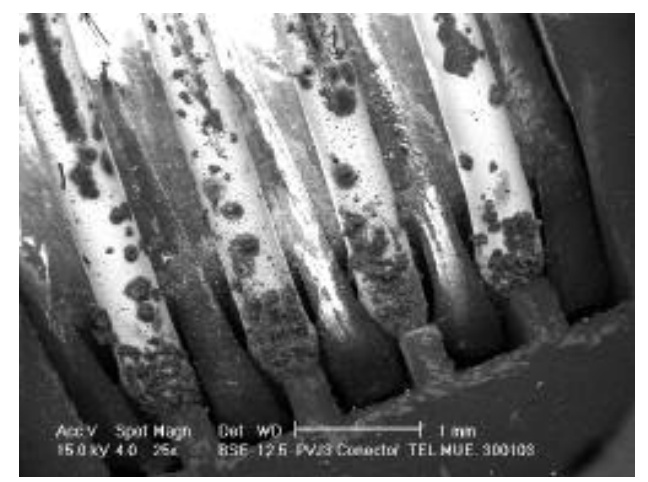




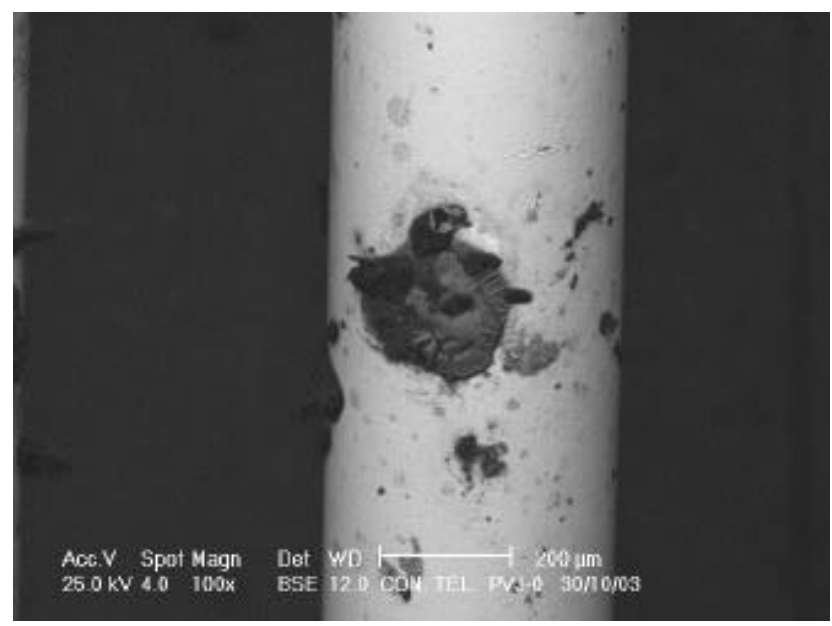

Figura 20. En un aparato telefónico existen diversos pines recubiertos de oro. La calidad del revestimiento no necesariamente es la misma, lo que se confirma a través del grado de deterioro

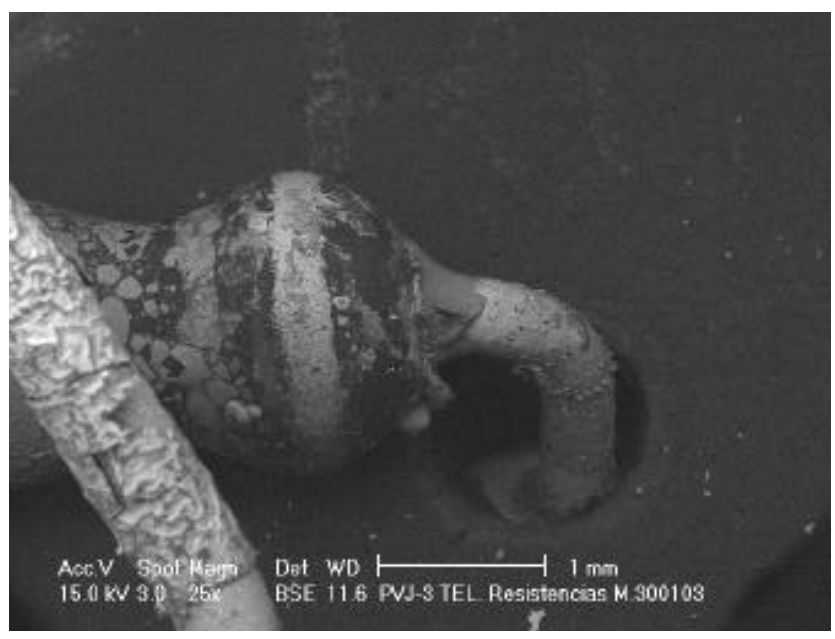

Figura 21. Conector de una resistencia. Se trata de un conductor de cobre recubierto con estaño. Los contaminantes presentes (cloruros) en combinación con una elevada humedad relativa dan como consecuencia un rápido deterioro del recubrimiento y su desprendimiento del conductor

mación de condensado, a diferencia de los de Tampico. En cuanto a los depósitos, se encontró también la presencia de cloruros y sulfuros de acuerdo a los materiales analizados, lo que permite suponer similares procesos a lo descrito para Tampico, con el énfasis de un menor deterioro.

Análisis de muestras provenientes de Bahía de Banderas (Puerto Vallarta)

En este caso, se tomaron muestras no solo del área del puerto sino de las regiones cercanas a Bahía de Banderas, las que se caracterizan por una intensa actividad agrícola y se encuentran prácticamente a nivel del mar a no más de 10 $\mathrm{km}$ de la costa.

Los aparatos correspondientes a la zona del puerto estuvieron en servicio en áreas con aire acondicionado, mientras que los de regiones agrí- colas operaron en sitios con amplio intercambio de aire con el ex te rior.

En gen eral, el deterioro es menor al correspondiente de las muestras provenientes de Tam- pico. La elaboración de los mapas de deterioro de las tarjetas (Figura 18) fue 
Cerrud-Sánchez S.M. †, Jacobo-Armendáriz V.H., Ortiz-Prado A. y Schouwenaars R.

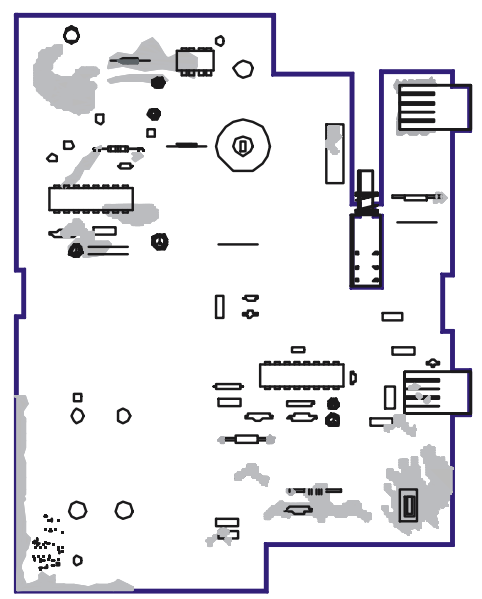

Figura 22. En las tarjetas correspondientes a aparatos que estuvieron en servicio en Cuernavaca, el deterioro fue mínimo. Sin embargo, en algunas de éstas se presentó una gran cantidad de depósitos, cuya apariencia permite considerar que su origen es antropogénico, lo que indica en este caso, que las fuentes de contaminación corresponden a dicho origen. La imagen es de un aparato retirado de

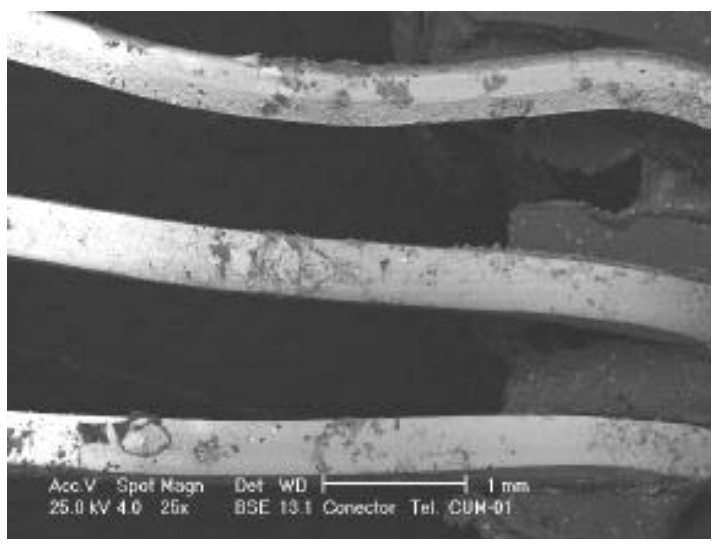

Figura 23. Conector de cobre recubierto de oro. El deterioro del recubrimiento es muy fuerte, existe un notable desprendimiento del mismo

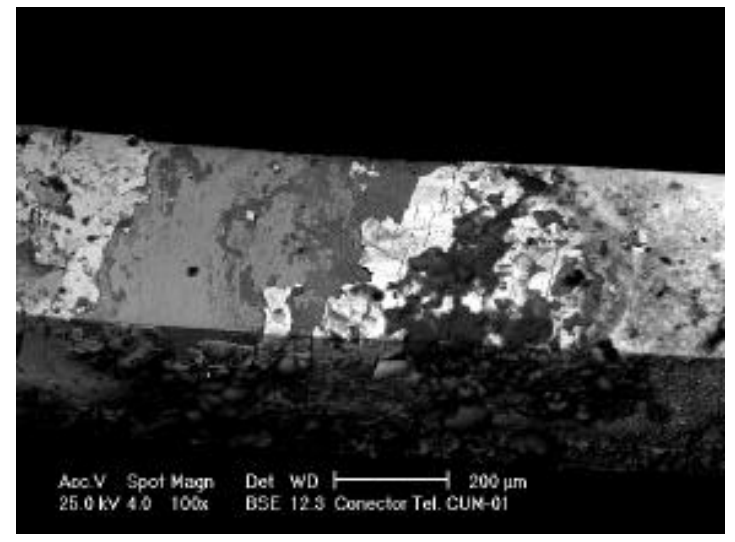

Figura 24. A la vez de desprenderse la película de oro se forman depósitos complejos en los cuales se evidencia 
Deterioro por corrosión atmosférica de componentes electrónicos de equipo telefónico

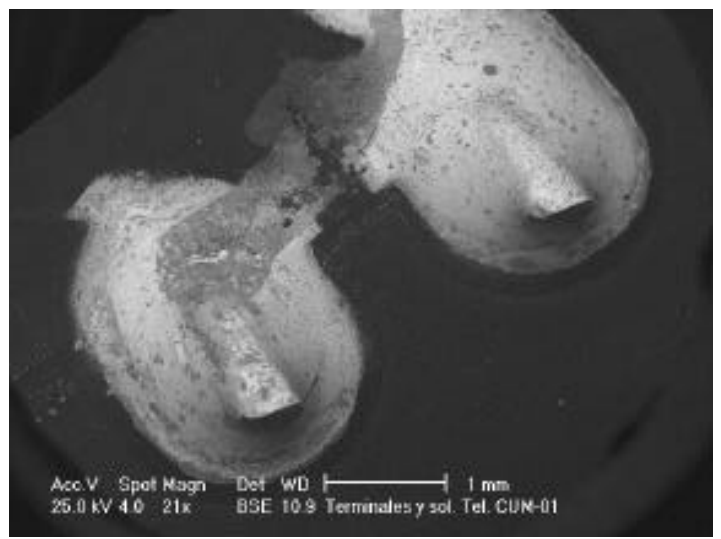

Figura 25. Región correspondiente a la unión de los conectores de las resistencias a las pistas de la tarjeta. Se trata de una soldadura SnPb, resulta evidente la presencia de residuos del fundente

compleja, ya que a simple vista resultó difícil determinar la presencia de zonas de condensado.

Para el análisis a detalle, se tomaron muestras de pines de conexión, resistencias e interruptores (Figura 19 a 21). El deterioro en conectores 0 en pines recubiertos con oro (Figuras 19 y 20) es mayor aún que lo observado en las muestras provenientes de Coatzacoalcos. En particular, el deterioro más severo se encuentra en los recu- brimientos de estaño (Figura 21). En todos los casos analizados, con excepción de un conector de oro, y a diferencia de las muestras provenientes de Tampico y Coatzacoalcos, no se encontró evidencia de la participación de azufre en el ata- que, identificando fundamentalmente la partici- pación de cloruros.

Se puede concluir que el deterioro se correla- ciona con la presencia de cloruros en combi- nación con la elevada humedad relativa carac- terística de la zona. Por otra parte, la región se caracteriza por una menor agresividad atmosférica (en cuanto a equipo electrónico), en comparación con la zona del Golfo.

\section{Análisis de muestras provenientes de Cuernavaca, Morelos}

La región correspondiente a Cuernavaca, Morelos, representa una zona de menor agresividad atmos- férica, ya que los principales contaminantes pro- vienen de fuentes móviles, así como aquellos característicos de las actividades agrícolas que se desarrollan en las regiones cercanas a la zona ur- bana. Adicionalmente se debe considerar el efecto de los productos propios de las actividades antropogénicas, ya que en este caso dichos con- taminantes pueden tomar un lugar preponderante en el deterioro. Esta hipótesis se confirma a partir del análisis de los contaminantes encontrados en algunas tarjetas y dispositivos (Figuras 22 a 25).

\section{Discusión}

La metodología empleada permite analizar el de- terioro que sufren los dispositivos bajo condi- ciones reales de exposición, esto en contraste con los datos obtenidos en pruebas de laboratorio. Los resultados permiten definir algunas modifi- caciones en el diseño y la manufactura para fa- vorecer el rendimiento del dispositivo como con- secuencia de evitar el deterioro ambiental. Asi- mismo, éstos representan una muestra evidente de la importancia que las condiciones climáticas y la agresividad del medio tropical o subtropical 
Cerrud-Sánchez S.M. †, Jacobo-Armendáriz V.H., Ortiz-Prado A. y Schouwenaars R.

tienen en comparación de los climas fríos y templados.

Del análisis de deterioro se constata la agresividad de las atmósferas interiores para un país como México, el cual se puede considerar como ejemplo típico de las condiciones económicas y de medio ambiente de las economías emergentes. Considerando los datos relativos a ventas de equipo de aire acondicionado, así como los de los censos económicos, se puede afirmar la limitada difusión en el empleo de estos equipos, y en consecuencia, confirmar que el nivel de contaminantes en interiores es del mismo orden que el de exteriores con la adición de los productos característicos de la actividad humana, así como producto de la descomposición de los diversos materiales que es común encontrar en interiores como parte inte gral de alfombras o cortinas.

El método propuesto para el análisis del deterioro en equipo electrónico no se puede catalogar como convencional. Sin embargo, es indis- cutible que ha permitido recabar información necesaria en cuanto al efecto de contaminantes y los problemas que se suscitan en campo.

En particular,el método planteado en el estudio no brinda detalles con relación a la naturaleza exacta de los mecanismos involucrados en el deterioro, pero sí proporciona información va- liosa con relación a la problemática y permite generar conclusiones que favorecen la definición de acciones preventivas, a la vez de precisar condi- ciones críticas de exposición. Por otra parte, estos análisis representan una primera aproximación en cuanto a los inconvenientes, que por corrosión se presentan en equipo electrónico, esto en las dife- rentes regiones climáticas características del país.

Si bien, el análisis se orientó a solo seis regiones del país, se considera que éstas son representativas de las diferentes atmósferas (ur-bana, rural, ma rina, in dus trial), a la vez que algunas de éstas, como en el caso de Coatzacoalcos y la Ciudad de México, son consideradas como críticas en cuanto a sus niveles de contaminación. Es por consecuencia que la información recabada per- mite tomar acciones de protección y mejora para el equipo instalado en condiciones similares a las de la exposición. No se realizaron estudios en zonas que se pudieran clasificar de muy baja corrosividad de acuerdo al tiempo de humectación (ISO 9223), situación que se valida a partir de los resultados obtenidos, ya que se constata que la humedad es variable fundamental en el deterioro del equipo electrónico.

El método propuesto es rápido, pues no fue necesario esperar los resultados de las pruebas de exposición (de hecho, las muestras empleadas representan en sí a los especimenes de expo- sición) para conocer la problemática característica de cada región, así como su grado de agresividad. La estimación rápida es una condición clave para este estudio, ya que a la vez de permitir la detección de problemas relacionados con el diseño y la manufactura, favorece también la definición de los agentes críticos para cada caso. Estos análisis son imposibles de realizar a partir exclusivamente de pruebas de laboratorio o de exposición de cupones metálicos, ya que la problemática bajo estudio resulta de la interacción de diversos factores (materiales, geometrías, condiciones de operación). Estas diferencias se traducen en la no coincidencia entre lo observado en campo y lo reportado de ensayos estándar. Es por consecuencia la importancia del presente enfoque, ya que permite relacionar de manera más directa los datos obtenidos con las necesidades de la in du stria electrónica.

Si bien, el enfoque experimentalseguido en el presente estudio está fuera de los procedimientos establecidos, éste ha permitido la determinación de los principales factores involucrados en la corrosión de los equipos electrónicos bajo las condiciones climáticas analizadas, es necesario entonces, mencionar que a la fecha no existen pruebas estándar que hayan podido llegar a los mismos resultados.

Se constata el efecto del empleo de sistemas de acondicionamiento de aire, esto en cuanto a la corrosividad característica del medio. Se deter- minó que la corrosión en interiores puede ser mucho más severa en 
Deterioro por corrosión atmosférica de componentes electrónicos de equipo telefónico

regiones tropicales y subtropicales que la característica a las zonas con menores niveles de humedad y temperatura. Re- sulta evidente, que si los altos niveles de conta- minación pueden influir en la rapidez del deterioro, éstos no representan la variable fundamental para el caso bajo estudio, sino que la humedad relativa resulta ser la vari able de mayor importancia en el deterioro del equipo electrónico, ya que además la presencia de condensado puede producir fallas intermitentes de los sistemas aún antes de que la corrosión origine daños.

Bajo estas condiciones, detalles de diseño que no son importantes en climas templados y secos, se convierten en factores vitales cuando el equipo es expuesto a climas húmedos y subhúmedos. De lo observado, se concluye la imperiosa necesidad de evitar al máximo la formación de celdas galvá- nicas, de mejorar la calidad de los revestimientos, sobre todo en el caso de los de oro. A través de los resultados del estudio, se comprueba que los recubrimientos de oro (sobre todo si son het erogéneos) son fuente importante de corrosión galvánica. Por otra parte, las zonas que provocan la acumulación de condensado y la formación de electrolitos, también deben ser eliminados. El recubrimiento con estaño y el empaque mediante polímeros son efectivos, con algunas excepciones, para retardar la presencia de inconvenientes por corrosión.

Asimismo, de este estudio se constata que las condiciones a las cuales se puede exponer el equipo son altamente variables dependiendo de las cir- cunstancias locales, pudiendo en ocasiones aproxi- marse a lo reportado para regiones templadas con atmósferas controladas, características de los países industrializados. Sin embargo, bajo la óptica de que más de la mitad de la población mundial vive en regiones con climas tropicales y subtropicales, se justifica la conveniencia de éste estudio y las conclusiones que de él se desprenden.

\section{Conclusiones}

El presente estudio ha permitido desarrollar una metodología para la evaluación del deterioro sufrido por el equipo electrónico al ser expuesto a diferentes condiciones climáticas. De los resul- tados se han podido comprobar las sustanciales diferencias que existen en el grado de ataque cuando se analizan muestras provenientes de regiones con diferencias climáticas, sobre todo en lo que se refiere a la humedad y temperatura. Se determinó que los altos índices de polución no influyen significativamente en la agresividad del ambiente, en lo que al equipo electrónico se refiere. Se ha constatado el efecto positivo que tiene el empleo de sistemas de acondicionamiento de aire, considerando que existe control de hume- dad, ya que en los equipos que se mantuvieron bajo dichas condiciones el deterioro fue insignifi- cante. Es por consecuencia que la exposición de equipo electrónico en climas tropicales y subtropicales representa una condición mas crítica que lo correspondiente a zonas templadas y secas, por lo que es necesario tomar las acciones preventivas adecuadas, desde el diseño del mismo, para evitar que se presenten fallas intempestivas de éstos, con las consecuencias negativas de cada caso.

\section{Agradecimientos}

Al Biólogo Germán Álvarez por su gran trabajo de inspección y análisis de muestras en el micros- copio electrónico de barrido (SEM), a los Inge- nieros Ignacio Cueva, Efraín Ramos y Roberto Cisneros por su apoyo en la preparación de mues- tras de tarjetas telefónicas; asimismo, agrade- cemos el apoyo económico brindado por la Direc- ción General de Asuntos del Personal Académico de la UNAM, a través del proyecto PAPIIT 119403-2.

\section{Referencias}

Abbott W. (1989). Corrosion of Electrical Contacts: Review of Flowing Mixed Gas Test Develop- ments. British Corrosion Journal, Vol. 14, 153.

Carrato A., Shaffer I. y Richwine R. (1978). Corrosion in Naval Aircraft Electronics Systems. Corrosion 78, National Associa tion of Corro sion Engi neers, NACE. 
Cerrud-Sánchez S.M. †, Jacobo-Armendáriz V.H., Ortiz-Prado A. y Schouwenaars R.

Cerrud S., Jacobo V., Ortiz A. y Schouwenaars R. (2002). Análisis de falla. Facultad de Ingeniería, UNAM.

Fukuda Y., et al. (1991). Indoor Corrosion of Copper and Silver exposed in Japan an Asian Countries. Journal of the Elec trochemicalSociety, Vol. 138.

García E. (1988). Modificaciones al sistema de clasificación climática de Koppen para adaptarlo a las condiciones de la

\section{Semblanza de los autores}

Sara Mercedes Cerrud-Sánchez †. Estudió la licenciatura de ingeniería mecánica eléctrica (área de ingeniería industrial) en la Facultad de Ingeniería de la UNAM, misma donde obtuvo el grado de maestra en ingeniería mecánica (2002). Fue miembro del personal académico de la FI-UNAM desde 1987, alcanzando el nivel de profesor titular "A" de tiempo completo definitivo en el área de manufactura y materiales. Participó en diversos proyectos y estudios para el sector público y privado, orientados al desarrollo y mejora de métodos de manufactura, corrosión y análisis de falla. Esta actividad se tradujo en más de 30 estudios y asesorías para la industria; asimismo, participó en 10 proyectos de investigación y desarrollo tecnológico, de los cuales tres fueron dirigidos por ella. Publicó cinco textos del área de manufactura, materiales y corrosión, así como cinco artículos en congresos internacionales, más de 35 artículos en congresos nacionales y ocho artículos en revistas arbitradas. Uno de sus últimos legados fue la definición e impulso de una línea de investigación en torno al modelado del comportamiento mecánico de hueso.

Armando Ortiz-Prado. Se graduó como ingeniero mecánico electricista en 1980 por la Facultad de Ingeniería de la UNAM, asimismo, obtuvo la maestría y el doctorado en ingeniería mecánica. Ha impartido cátedra desde hace 25 años en las áreas de procesos de manufactura y ciencia de materiales. Actualmente, es tutor doctoral de los posgrados de ciencia e ingeniería de materiales y de ingeniería. Sus líneas de investigación son modelado de procesos de conformado mecánico, análisis de falla, comportamiento mecánico de biomateriales y corrosión en equipo electrónico. Ha desarrollado proyectos y estudios para diferentes empresas privadas y entidades gubernamentales, orientados al desarrollo y mejora de métodos de manufactura, desarrollo de materiales, corrosión y análisis de falla; lo cual se traduce en más de 50 estudios y asesorías para la industria, 10 proyectos de investigación y desarrollo tecnológico. A la fecha, suma más de 70 tesis de licenciatura concluidas, así como 6 de maestría y una de doctorado. Su experiencia se ha transmitido a través de 6 textos para licenciatura, esto en las áreas manufactura, materiales y corrosión. Asimismo, su productividad incluye 15 artículos en congresos internacionales, más de 50 artículos en congresos nacionales y más de 10 artículos en revistas arbitradas. Fue galardonado por la UNAM con la Distinción Universidad Nacional para jóvenes académicos (Docencia en Ciencias Exactas) en 1989. Recibió la Cátedra espe cial "Mariano Hernández Barrenechea" de Facultad de Ingeniería UNAM en 1997 y 1998. Su productividad académica y científica ha sido reconocida por la UNAM a través del otorgamiento del nivel D del PRIDE y por el CONACYT con el nombramiento de investigador nacional.

Víctor Hugo Jacobo-Armendáriz. Es ingeniero mecánico electricista en el área de ingeniería indus trial, egresado de la Facultad de Ingeniería, UNAM, institución donde también obtuvo los grados de maestro y doctor en ingeniería. Actualmente, funge como profesor en el área de manufactura y materiales, en la propia FI-UNAM. Su línea de investigación se ha orientado al análisis de falla, la ciencia de materiales y los sistemas expertos (tema en el cual obtuvo su doctorado). De 10 años a la fecha ha participado en diversos proyectos y estudios para el desarrollo y mejora de métodos de manufactura, corrosión y análisis de falla. Ha dirigido 6 tesis de licenciatura y publicado 2 textos en (análisis de falla, corrosión y protección), 4 artículos en congresos internacionales, más de 25 artículos en congresos nacionales y 6 artículos en revistas arbitradas. Recibió la Cátedra Espe cial SEFI en 2001 y la Antonio Dovalí en 2002.

Rafael Schouwenaars-Franssens. Obtuvo el grado de maestro en ingeniería de minas en la Universidad de Lovaina,

Bélgica. Durante cuatro años estuvo activo como investigador en varios proyectos relacionados con el estudio de la textura cristalográfica en aceros a bajo carbono en el Departamento de Metalurgia e Ingeniería de Materiales de la misma universidad. Su trabajo en México lo inició como experto en microscopía electrónica dentro de la Unidad de Investigación y Asistencia Tecnológica en Materiales (UDIATEM). Posteriormente, combinó estas actividades con una carrera de profesor en la Facultad de Ingeniería. Obtuvo el doctorado en ingeniería mecánica en al año 2004, y fue reconocido como investigador nacional (nivel I) en el 2005. En el ámbito tecnológico se dedica al análisis de falla y la optimización de procesos en la indu stria metal-mecánica. Esto ha llevado a una serie de estudios científicos 
República Mexicana, 4a edición. Instituto de Geografía, UNAM.

Gouda V.K., Carew J.A. y Riad W.T. (1989). Investigation of Computer Hardware Failure due to Corrosion. British. Corrosion Journal, Vol. 24, 192.

Guttenplan J.D. (1987). Corrosion in the Electronics Industry. Metals Handbook, ASMInternational, Vol. 13.

INE-CENICA-JICA (1997). Segundo informe sobre la calidad del aire en ciudades mexicanas (datos de 1995).

Instituto Nacional de Estadística, Geografía e Informática (INEGI) (2003). Información geográfica.

Mariaca R.L., Genesca J. y Uruchurtu Ch.J. (1999). Corrosividad atmosférica. Plaza y Valdez Editores- UNAM.

Oesh S. y Faller M. (1997). Environmental Effects on Mate rials: The Effect of the Air Pollutants $\mathrm{SO}_{2}, \mathrm{NO}_{2}, \mathrm{NO}$ and $\mathrm{O}_{3}$ on the Corro sion of Copper, Zinc and Aluminium a Short Literature Survey and Results of Laboratory Exposures. Corrosion Science, Vol. 39, 105.

Rice D.W., Cappell R.J., Kinsolving W. y Laskowski J. (1980). Indoor Corrosion of Metals. Journal of the Electrochemical Society, Vol.127.

UNEP/WHO (1992). Urban Air Pollution in Megacities of the World.

White E., Slensky G. y Dobbs B. (1987). Case Histories and Failures of Electronics and Communications Equipment. Metals Handbook, ASM International, Vol. 13.
Yukonovich (2001). Electronics CC Technologies Laboratories Inc . Dublín Ohio.

Zakipour S. y Leygraf C. (1986). Evaluation of Laboratory Tests to Simulate Indoor Corrosion of Electrical Contact Materials. Journal of the Electrochemical Society, Vol. 133, 21.

Zakipour S. Tidblad J. y Leygraf C. (1997). Atmospheric Corrosion Effects of $\mathrm{SO}_{2}$, $\mathrm{NO}_{2}$, and $\mathrm{O}_{3}$. Journal of the Elec tro chemical Society, Vol. 144,3513. 